\title{
High-Coherence Fluxonium Qubit
}

Long B. Nguyen, Yen-Hsiang Lin, Aaron Somoroff, Raymond Mencia, Nicholas Grabon, and Vladimir E. Manucharyan Department of Physics, Joint Quantum Institute, and Center for Nanophysics and Advanced Materials, University of Maryland, College Park, Maryland 20742, USA

(Received 6 November 2018; revised manuscript received 15 September 2019; published 25 November 2019)

\begin{abstract}
We report superconducting fluxonium qubits with coherence times largely limited by energy relaxation and reproducibly satisfying $T_{2}>100 \mu \mathrm{s}\left(T_{2}>400 \mu \mathrm{s}\right.$ in one device). Moreover, given the state-of-the-art values of the surface loss tangent and the $1 / f$ flux-noise amplitude, the coherence time can be further improved beyond $1 \mathrm{~ms}$. Our results violate a common viewpoint that the number of Josephson junctions in a superconducting circuit—over $10^{2}$ here—must be minimized for best qubit coherence. We outline how the unique to fluxonium combination of long coherence time and large anharmonicity can benefit both gate-based and adiabatic quantum computing.
\end{abstract}

DOI: 10.1103/PhysRevX.9.041041

Subject Areas: Mesoscopics, Quantum Information, Superconductivity

\section{INTRODUCTION}

Quantum superconducting circuits based on Josephson tunnel junctions have become a leading platform in the pursuit of quantum computing [1]. These artificial "atoms" can be printed on a chip in large numbers, wired together for strong interactions, and precisely manipulated and read out by radio-frequency electronics [2]. The Josephson tunnel junction provides the necessary nondissipative nonlinearity required to turn linear electrical circuits into quantum bits (qubits) and strong circuitcircuit coupling into fast logical operations. The weak point of superconducting qubits is their relatively short coherence times. Decoherence introduces errors during gate operations [3] and constrains the number of qubits that can coherently tunnel in a quantum annealer [4]. With the growing interest in complex quantum processors [5-9], improving intrinsic coherence of superconducting qubits without sacrificing their controllability remains a central problem.

Material imperfections, in the form of dielectric loss and $1 / f$ flux noise, are the major obstacles in improving the coherence of superconducting qubits. The former effect is believed to be due to microscopic two-level charge defects residing in the interface oxide layers of a typical thin-film device $[10,11]$. As a consequence, each circuit capacitance acquires a nonzero dielectric loss tangent which induces the energy relaxation of the qubit. The latter effect is likely due

Published by the American Physical Society under the terms of the Creative Commons Attribution 4.0 International license. Further distribution of this work must maintain attribution to the author(s) and the published article's title, journal citation, and DOI. to unpaired electrons trapped in the same oxide layers and acting as spin- $1 / 2$ impurities. Their low-temperature dynamics generates a noisy magnetic flux through any superconducting loop with a $1 / f$-type spectral density [12]. Hence, a flux-tunable qubit generally comes at the expense of dephasing. In the case of conventional flux qubits, the dephasing time rapidly drops to a few nanoseconds upon detuning from the half-integer flux bias $[13,14]$. Furthermore, recent studies suggest that flux noise can also induce energy relaxation of flux qubits, presumably through the absorption of GHz-frequency photons by spin defects $[15,16]$.

Upgrading circuit materials is challenging [17,18]. Alternatively, coherence can be improved by designing noise-insensitive circuits. The successful tricks so far all sacrifice qubit anharmonicity. For example, a transmon qubit is derived from a Cooper pair box by shunting the junction with a large external capacitance [19]. The qubit sensitivity to $1 / f$ charge noise dropped exponentially, but the spectrum of the circuit also evolved from that of a nearly perfect two-level system to that of an oscillator with about 5\% anharmonicity. Capacitive shunting of a flux qubit helps reduce its unnecessarily large flux sensitivity but with a similar reduction of anharmonicity [20]. The low anharmonicity adds more challenges to scaling. Since nonlinearity enables logic, reducing it too much will inevitably slow down gate operations. For example, the dispersive shift in circuit quantum electrodynamics (circuit QED) drops [19] with the qubit-oscillator detuning $\Delta$ much faster for a transmon $\left(1 / \Delta^{2}\right)$ than for the charge qubit $(1 / \Delta)$. Another problem is that exciting multiple qubits creates nearly resonant conditions for state leakage outside of the computational subspace, an error which is difficult to correct algorithmically. 
In this work, we describe fluxonium [21] superconducting qubits (Fig. 1) designed to evade decoherence due to dielectric loss and flux noise without sacrificing anharmonicity, flux-tuning range, or controllable interactions. The circuit design is identical to that introduced in a recent work [22], where an energy relaxation time in the milliseconds range was demonstrated by operating fluxoniums away from both an integer and a half-integer flux bias. Here the devices are studied near the half-integer flux bias, where the transition frequency reaches the minimal value and its first-order sensitivity to external flux vanishes. We observe $T_{2}>100 \mu$ s in eight devices with varying circuit parameters. One device has $T_{2}>400 \mu \mathrm{s}$, another device has $T_{2}>300 \mu \mathrm{s}$. These record-long coherence times are still largely limited by energy relaxation. Frequency dependence of relaxation times $T_{1}$ suggests that this process is dominated by the surface loss around the large external antenna shunting the weak junction. Thus, even longer coherence times are expected on upgrading our fabrication procedures to the state of the art.

This paper is organized as follows. In Sec. II we introduce fluxonium. In Sec. III we describe measurements of nine devices labeled $A-I$ (see Table I). In Sec. IV we discuss the data in the context of known decoherence channels. Section V offers a perspective on utilizing lowfrequency fluxonium qubits in the existing quantumcomputing schemes. Section VI concludes the work.

\section{FLUXONIUM}

A fluxonium circuit consists of a Josephson junction with energy $E_{J}$ shunted by a capacitance $C$ and an inductance $L$ [Figs. 1(a) and 1(b)]. The two linear elements introduce the charging energy $E_{C}=e^{2} / 2 C$ and the inductive energy $E_{L}=(\hbar / 2 e)^{2} / L$. The parameters must satisfy $E_{L} \ll E_{J}$ and $1 \lesssim E_{J} / E_{C} \lesssim 10$, which distinguish fluxonium from other inductively shunted junction devices. These conditions place a challenging requirement on the value of $E_{L}$, which translates to an extremely large inductance per unit length of about $10^{4} \mu_{0}$, where $\mu_{0}$ is the vacuum permeability. To meet this requirement, the inductance $L$ is constructed from the kinetic inductance of a tightly packed chain of $N \approx 10^{2}$ moderate-area (approximately $1 \mu \mathrm{m}^{2}$ ) Josephson tunnel junctions [Fig. 1(c)]. One can interpret fluxonium as a transmon where the weak junction is short circuited at low frequencies, and hence, there is no sensitivity to offset charges even with $E_{J} / E_{C} \sim 1$. Consequently, there is no need for a large shunting capacitance, and hence, circuit anharmonicity can be large. One can also view fluxonium as a generalized $N$-junction flux qubit, where the first- and second-order coupling to flux noise is suppressed as $1 / N$ and $1 / N^{2}$, respectively, without significantly reducing the frequencytuning range. The circuit Hamiltonian is [23]

$$
H=4 E_{C} n^{2}+\frac{1}{2} E_{L} \phi^{2}-E_{J} \cos \left(\phi-\phi_{\mathrm{ext}}\right),
$$

where $\phi$ is the phase twist across the inductance, and $2 e \times n$ is the displacement charge at the capacitance. The two operators obey $[\phi, n]=i$. The quantity $\phi_{\mathrm{ext}}$ is the reduced magnetic flux biasing the loop formed by the weak junction and the shunting inductance. Near $\phi_{\text {ext }}=0$, the low-energy spectrum corresponds to plasmalike oscillations in the central Josephson well with frequencies and transition dipoles similar to those of a transmon [Fig. 1(d)]. (a)

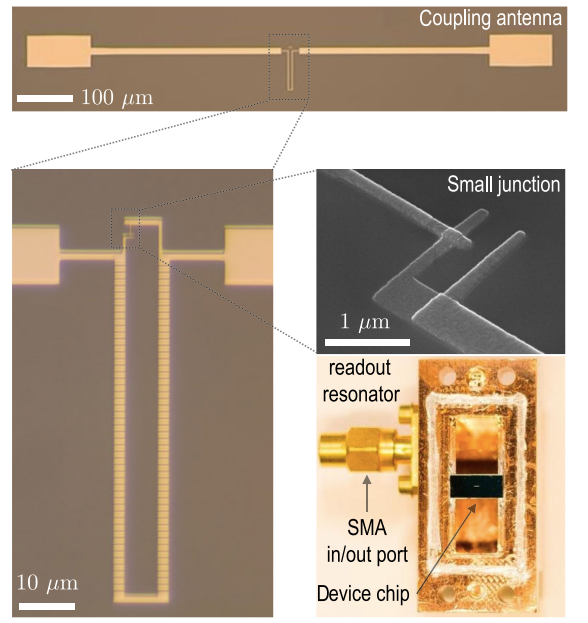

(b)

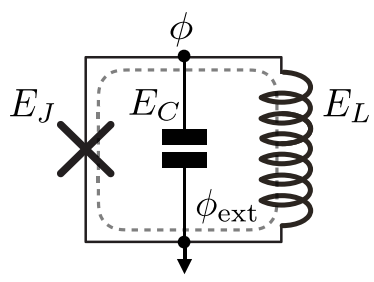

(d)

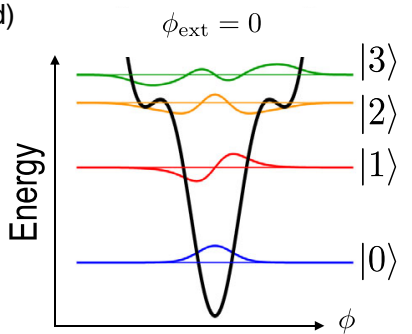

(c)

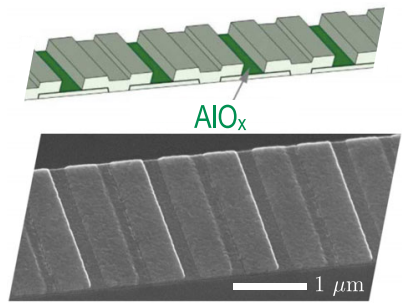

(e)

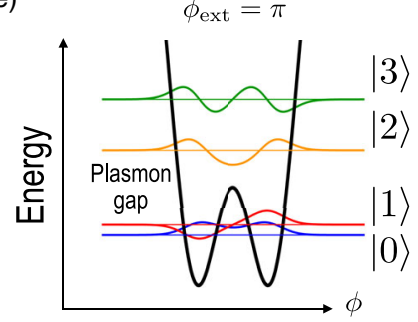

FIG. 1. (a) Images of a single-junction fluxonium device indicating the antenna, the loop, the small junction, and the chip mounted in a copper resonator. (b) The three-element circuit model of fluxonium. (c) Implementation of a large-value inductance $L$ using a linear chain of Josephson junctions. (d),(e) The particle-in-a-box potential energy, the spectrum, and the eigenstates for the circuit model in (b) in the cases $\phi_{\text {ext }}=0$ (d) and $\phi_{\text {ext }}=\pi(e)$, which is the focus of the present work. 
Here we operate the qubit near the $\phi_{\mathrm{ext}}=\pi$ "sweet spot," where the first-order sensitivity of the transition frequency to flux vanishes by symmetry [Fig. 1(e)]. The qubit states $|0\rangle$ and $|1\rangle$ correspond to the tunnel splitting of the twofold degenerate classical ground state. The circuit parameters are such that the qubit transition frequency is about an order of magnitude lower than that at $\phi_{\text {ext }}=0$. The noncomputational states $|2\rangle,|3\rangle$, etc., are separated by a plasmon gap [Fig. 1(e)], and they form an anharmonic spectrum with a rich selection rule structure.

By reducing the qubit frequency tenfold from the typical (4-8)-GHz range, the energy relaxation rate is expected to naturally slow down without the need for improving materials. One might think this trick would inevitably slow down interactions too, as it happens with weakly anhamonic oscillators. Fortunately, this is not the case with fluxoniums. For example, the dispersive coupling to a cavity mode can be as large as it is in a typical transmon setup owing to the high frequencies and dipoles of transitions outside the computational space $[22,24,25]$. These transitions were recently utilized to theoretically demonstrate a high-fidelity microwave-activated Controlled-Z (CZ) gate between two capacitively or inductively coupled fluxoniums [26]. Furthermore, inductive coupling can be made large even within the computational subspace, as it was experimentally demonstrated by the molecular binding spectra of two fluxoniums with shared chain junctions [27].

The devices reported in this work spectacularly break the old unwritten rule of superconducting qubits design: For the best coherence, the number of Josephson junctions per qubit should be minimized. On the one hand, this rule is motivated by the belief that the $\mathrm{Al} / \mathrm{AlOx} / \mathrm{Al}$ Josephson tunnel junction is likely the faultiest part of a qubit circuit, and hence, the probability for a fatal failure might quickly grow with the number of junctions. On the other hand, this rule severely limits the design options for inventing superior quantum hardware. For example, the minimal number $N=3$ is deliberately chosen in creating the threejunction flux qubit [28], which results in an impractically high sensitivity to external flux $[13,14]$. Unfortunately, the lack of high coherence in previously measured fluxoniums [24,27,29] and other multijunction qubits [30-32] further contributed to discouraging the exploration of complex superconducting circuits. The main deficiency of earlier studies was a small number (typically one or two) of tested devices. This prevents us from knowing with certainty what specific problems are fixed in the present work. Instead, our goal is to share a concrete fluxonium design that yields high-coherence times reproducibly over many devices.

\section{EXPERIMENT}

Similar to the original fluxonium design [21], here we attach an external capacitance in the form of a simple dipolar antenna directly to the small junction [Fig. 1(a)]. The capacitance in Fig. 1(b) is mainly due to this antenna. Device $C$ (Table I) contains a split-junction (dc superconducting quantum-interference device). It is measured at the simultaneous sweet spot (the so-called double sweet spot) of both loops, which are fabricated to have commensurate areas. In all other devices we use a single junction for simplicity. The qubits are capacitively coupled to a 3D copper box readout mode [Fig. 1(a)] with a frequency of $7.5 \mathrm{GHz}$ and a linewidth $\kappa / 2 \pi \approx 15 \mathrm{MHz}$ (this is equivalent to a rather low external quality factor $Q=500$ ). The state of the qubit is monitored in a basic two-port cavity transmission measurement. For consistency, all devices are measured at a small external magnetic field. The precise effect of this field remains inconclusive at this stage. Details of the cryogenic setup [Fig. 5] are provided in the Appendix.

The devices are fabricated in a single step using the fourdecade-old Dolan bridge technique [33,34]. This method is extremely robust for a typical chain junction area of about $1 \mu \mathrm{m}^{2}$. It also yields the smallest possible stray capacitance in a planar geometry and up to (30000-40 000)-junctionlong chains can be fabricated without a single fault $[35,36]$. A sketch and an image of a section of a typical fluxonium chain is shown in Fig. 1(c). The advantage of our chain

TABLE I. Summary of relevant device parameters. The values of $E_{J}, E_{C}, E_{L}$ are obtained from spectroscopy fits; the qubit dispersive shift $\chi_{01}$ is calculated using data and formulas of Ref. [22]; loss mechanism estimates are discussed in Sec. IV.

\begin{tabular}{|c|c|c|c|c|c|c|c|c|c|c|c|c|c|}
\hline Qubit & $\begin{array}{c}E_{J} \\
(\mathrm{GHz})\end{array}$ & $\begin{array}{c}E_{C} \\
(\mathrm{GHz})\end{array}$ & $\begin{array}{c}E_{L} \\
(\mathrm{GHz})\end{array}$ & $N$ & $\begin{array}{c}T_{1} \\
(\mu \mathrm{s})\end{array}$ & $\begin{array}{c}T_{2} \\
(\mu \mathrm{s})\end{array}$ & $\begin{array}{c}\omega_{01} / 2 \pi \\
(\mathrm{GHz})\end{array}$ & $\omega_{12} / \omega_{01}$ & $\begin{array}{c}\chi_{01} / 2 \pi \\
(\mathrm{MHz})\end{array}$ & $\begin{array}{c}\tan \delta_{C} \\
\left(\times 10^{-6}\right)\end{array}$ & $\begin{array}{r}\tan \delta_{\mathrm{AlOx}} \\
\left(\times 10^{-4}\right)\end{array}$ & $\begin{array}{c}x_{\mathrm{QP}} \\
\left(\times 10^{-8}\right)\end{array}$ & $\begin{array}{c}\tan \delta_{L} \\
\left(\times 10^{-8}\right)\end{array}$ \\
\hline$A$ & 3 & 0.84 & 1 & 100 & 110 & 160 & 0.78 & 3.4 & 0.27 & 1.7 & 1.1 & 3.84 & 15.4 \\
\hline$B$ & 4.86 & 0.84 & 1.14 & 136 & 250 & 150 & 0.32 & 11.1 & 0.57 & 1.5 & 1.3 & 0.52 & 2.03 \\
\hline$C$ & $2.2^{\mathrm{a}}$ & 0.55 & 0.72 & 102 & 260 & 350 & 0.48 & 3.8 & 0.08 & 1.23 & 0.9 & 1.77 & 5.75 \\
\hline$D$ & 2.2 & 0.83 & 0.52 & 196 & 70 & 90 & 0.56 & 4.1 & 0.1 & 3.12 & 4.0 & 7 & 28.25 \\
\hline$E$ & 1.6 & 0.86 & 0.5 & 100 & 108 & 140 & 0.83 & 2.5 & 0.05 & 1.52 & 1.0 & 7.8 & 30.22 \\
\hline$F$ & 3.4 & 0.8 & 0.41 & 348 & 270 & 165 & 0.17 & 18.3 & 0.28 & 1.94 & 4.5 & 0.63 & 2.1 \\
\hline$G$ & 1.65 & 1.14 & 0.19 & 400 & 110 & 140 & 0.55 & 4.1 & 0.03 & 2 & 3.8 & 8.65 & 34.9 \\
\hline$H$ & 4.43 & 1 & 0.79 & 100 & 230 & 235 & 0.32 & 11.8 & 0.1 & 1.68 & 0.9 & 0.72 & 2.85 \\
\hline$I$ & 3.43 & 1 & 0.58 & 144 & 500 & 510 & 0.395 & 8.2 & 0.08 & 0.63 & 0.5 & 0.53 & 2.11 \\
\hline
\end{tabular}

${ }^{\mathrm{a}}$ Effective $E_{J}$ of the split junction biased by a half-integer flux. 


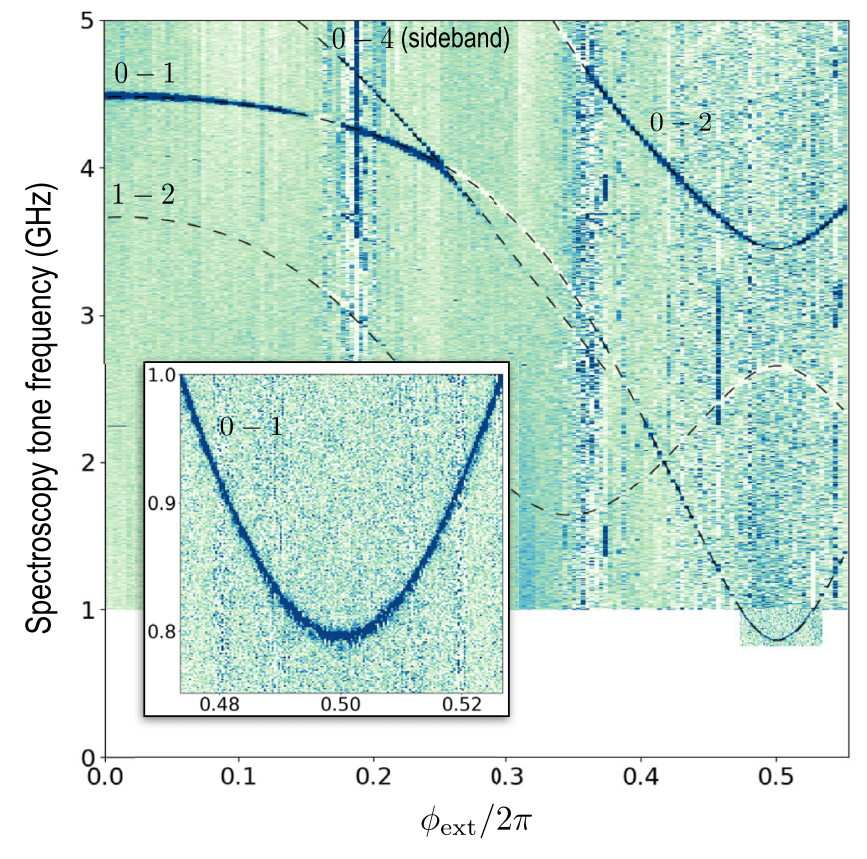

FIG. 2. Two-tone spectroscopy transmission signal (arbitrary units) as a function of the spectroscopy frequency and flux through the loop for device $A$. The contrast is optimized piecewise to maximize the visibility of the resonances. Lines indicate a fit to the spectrum of the Hamiltonian (1). The extra resonance line crossing (not anticrossing) the qubit transition $0-1$ is the red sideband of the $0-4$ transition and the readout at $7.5 \mathrm{GHz}$.

geometry is that it does not require precision tuning of the resist mask. For the substrate, we use high-resistivity silicon covered by a native oxide. The fabrication procedure is described in the Appendix.

The measured spectrum of device $A$ together with the fitted theory lines are shown in Fig. 2. The data are obtained by conventional two-tone spectroscopy [37]. The theory is a result of numerical diagonalization of the Hamiltonian (1) with $E_{J}, E_{C}, E_{L}$, and flux-to-coil current conversion as fit parameters. Note that in addition to fitting the two lowest frequency transitions, the theory precisely matches the red sideband of the readout mode with the transition between states $|0\rangle$ and $|4\rangle$. This indicates that there are no stray chain modes at frequencies below $10 \mathrm{GHz}$, and the Hamiltonian (1) is an accurate model of our complex device. At $\phi_{\text {ext }}=0$, the qubit transition (labeled 0-1) corresponds to anharmonic oscillations in the central Josephson well. The nature of this transition, along with its frequency at about $4.5 \mathrm{GHz}$ and the value of the transition dipole $\langle 0|\phi| 1\rangle$, is similar to that of a typical transmon qubit. The difference here is that the Josephson well is slightly deformed by the shunting inductance [Fig. 1(c)] and the anharmonicity approximately given by $E_{C} \approx 0.8 \mathrm{GHz}$, is considerably enhanced owing to the reduced value of the shunting capacitance.

As the flux is tuned toward a sweet spot at $\phi_{\text {ext }}=\pi$, the qubit transition monotonically drops to about $800 \mathrm{MHz}$.
Already with the naked eye, it is evident that the sensitivity of the qubit transition to flux does not exceed about $20 \mathrm{GHz}$ per flux quantum, while the frequency is tuned by over two octaves. Because of the thermal occupation of the $|1\rangle$ state, one can see the transition 1-2 in the small vicinity of $\phi_{\text {ext }}=\pi$. The transition $0-2$ is parity forbidden exactly at $\phi_{\text {ext }}=\pi$, which is correctly reflected by the continuous reduction of its power-broadened linewidth (in a fixedpower experiment) upon tuning the flux toward the sweet spot. The large anharmonicity of the qubit at the sweet spot can be characterized by the ratio $\omega_{12} / \omega_{01} \approx 3-10$ for our typical circuit parameters. Finally, we note that no twolevel defects are spotted in the spectrum near $\phi_{\mathrm{ext}}=\pi$ with anticrossings larger than a few MHz (Fig. 2, inset).

The frequency dependence of energy relaxation time $T_{1}$ covering several frequency octaves is measured by flux tuning the qubit transition between $\phi_{\mathrm{ext}}=0$ and $\phi_{\mathrm{ext}}=\pi$. The $T_{1}$ values are obtained by a standard time-domain experiment recording the evolution of the cavity transmission following a $\pi$ pulse to a qubit. The majority of relaxation signals fit well to an exponential function, and the characteristic decay time is quoted as $T_{1}$. We observe infrequent instances of a double-exponential decay, and those cases are not included in extracting the qubits' $T_{1}$ values.

To extract the noise spectral density $S_{\phi}(\omega)$, we normalize the measured relaxation lifetimes by the square of the matrix element and plot the quantity $T_{1} \times|\langle 0|\phi| 1\rangle|^{2}$ as a function of the qubit frequency $\omega_{01} / 2 \pi$ (Fig. 3). According to Fermi's golden rule [38], this quantity is inversely proportional to the spectral density of the noise coupled to the phase variable $\phi$ at the qubit frequency $\omega$ [see Eqs. (A5)-(A7)]. At frequencies above $1 \mathrm{GHz}$, the data follow a simple model $S_{\phi}(\omega)=\hbar C \omega^{2} \tan \delta_{C}$, assuming an effective frequency-independent dielectric loss tangent $\tan \delta_{C}$ of the total capacitance $C$ across the weak junction. At lower frequencies, we have to account for the stimulated emission by thermal photons in the environmental modes and a possible frequency dependence of the dielectric loss tangent. The later effect is modeled empirically [see Eq. (A8)] as $\tan \delta_{C} \propto \omega^{\epsilon}, \epsilon>0$. Using $T=20 \mathrm{mK}$ (base temperature of the dilution refrigerator), $\epsilon=0.15$, and an interval $\tan \delta_{C}(\omega=2 \pi \times 6 \mathrm{GHz})=(2.0-3.6) \times 10^{-6}$, we obtain a good agreement between theory [Eqs. (A9) and (A10)] and the measured $T_{1}$ times for all devices biased at their sweet spots (Fig. 3, bottom).

The coherence time measurement is performed using a standard single $\pi$-pulse echo sequence [13] in order to eliminate the uninformative low-frequency (minute-scale) drifts in the setup. This protocol choice is also dictated by the relatively low readout efficiency in this particular setup, which requires minutes of averaging time. The measured coherence times $T_{2}$ as a function of the flux for device $A$ are shown in Fig. 4(a). Away from the sweet spot, where the qubit transition is maximally sensitive to flux, we typically 


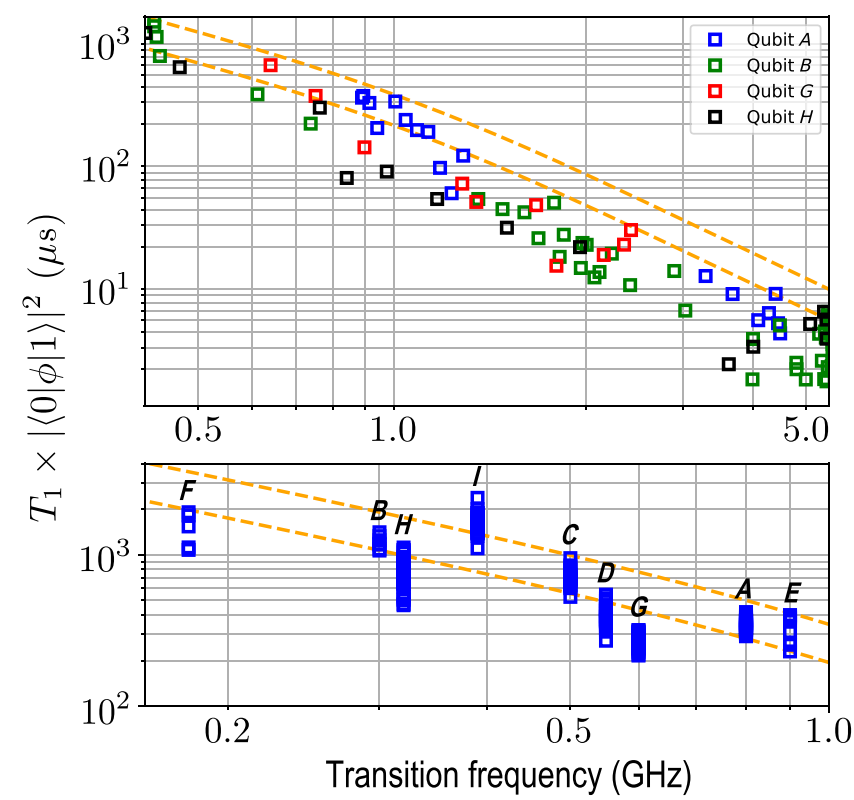

FIG. 3. (Top) Normalized energy relaxation time (a quantity inversely proportional to the spectral density of noise coupled to the $\phi$ variable) as a function of the qubit transition frequency measured by tuning flux in devices $A, B, G, H$. (Bottom) Same quantity, including repeated in time measurements, for all devices $A-I$ biased at their half-integer flux sweet spots. Dashed lines represent a dielectric loss theory (see text).

measure $T_{2} \approx 3-6 \mu \mathrm{s}$, and the echo signal has a distinct Gaussian shape [Fig. 4(b), top]. This result confirms that decoherence is due to pure dephasing. Tuning the flux toward the sweet spot, we observe a monotonic rise in the coherence time to about $T_{2} \approx 100 \mu \mathrm{s} \gtrsim T_{1}$. Here the echo signal is exponential, which is consistent with relaxationdominated decoherence [Fig. 4(b), bottom]. The flux dependence of $T_{2}$ agrees with the first-order coupling to the $1 / f$ flux noise with the amplitude $2 \times 10^{-6}(h / 2 e) / \sqrt{\mathrm{Hz}}$ at $1 \mathrm{~Hz}$.

To understand the robustness of the measured $T_{2}$ values at the sweet spot, we perform repeated interleaved measurements of times $T_{1}$ and $T_{2}$. The results for the representative devices $A, C$, and $I$ are shown in Fig. 4(c). Each attempt takes approximately $30 \mathrm{~min}$ of data acquisition. Both values of $T_{1}$ and $T_{2}$ drift in time, but their variation typically does not exceed a factor of 2 over about a half-day. In some attempts we observe $T_{2} \approx 2 T_{1}$, but a typical situation is $T_{1}<T_{2}<2 T_{1}$. We believe that the difference between $2 T_{1}$ and $T_{2}$ may be caused by a combination of imperfect tuning of echo pulses, possible flux drifts due to imperfect magnetic shielding, and long averaging times. However, fluctuations in pure dephasing time, formally defined as $1 /\left(1 / T_{2}-1 / 2 T_{1}\right)$ cannot be fully excluded at this stage. A setup involving a quantum-limited amplifier will clarify the details of the temporal drift of coherence times in future experiments. However, it is clear that the coherence is already largely limited by energy relaxation. The longest reliably measured values of $T_{2}$ together with the accompanying value of $T_{1}$ are quoted for all devices in Table I. Indeed, the worst (best) coherence device $D(I)$ has $T_{2}=90 \mu \mathrm{s}\left(T_{2}>400 \mu \mathrm{s}\right)$ and corresponds to a slightly lower (higher) than usual value of $T_{1}$ (Table I). Interestingly, the second longest coherence time $\left(T_{2}>300 \mu \mathrm{s}\right)$ is observed in device $C$, which contains a split junction, and it is thus sensitive to flux noise in two loops. This strengthens the conclusion that flux noise is not a limiting decoherence mechanism in our devices at the sweet spot. (a)

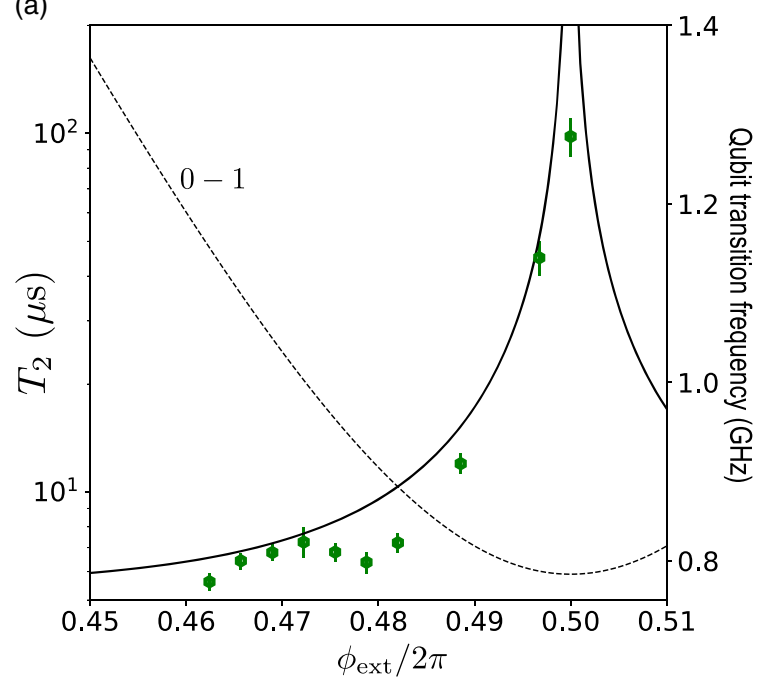

(b)

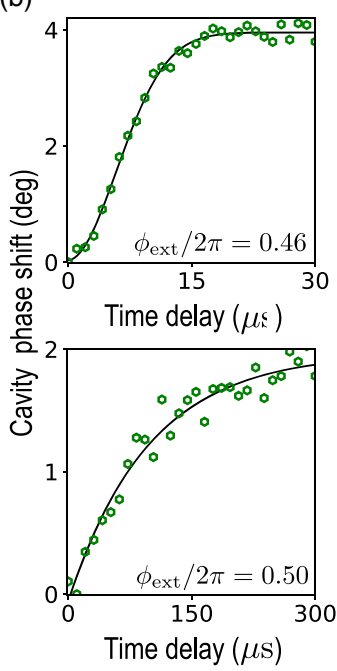

(c)

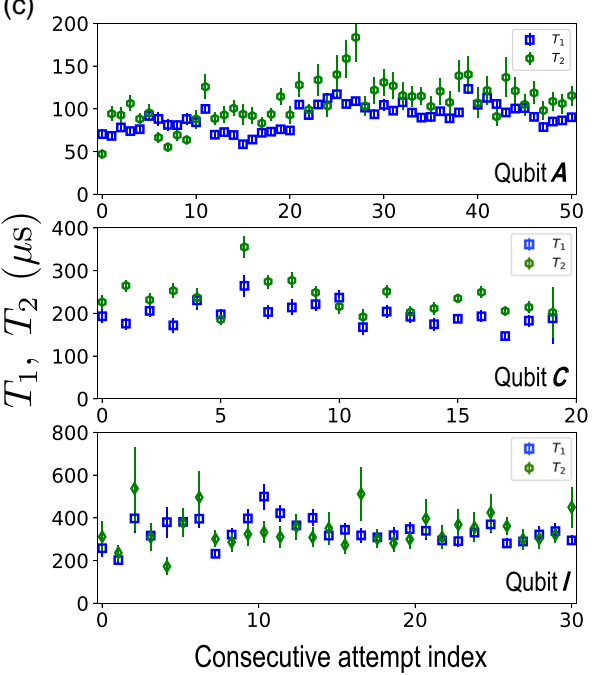

FIG. 4. (a) Coherence time $T_{2}$ (markers) and the qubit frequency (dashed line) as a function of the flux. Solid line indicates a prediction for the first-order coupling to a $1 / f$ flux noise. (b) Gaussian echo signal away from the sweet spot (top) and an exponential echo signal at the sweet spot (bottom). (c) Interleaved measurement of temporal fluctuations of $T_{1}$ (blue markers) and $T_{2}$ (green markers) at $\phi_{\text {ext }}=\pi$ over a time interval of approximately $12 \mathrm{~h}$. 


\section{DISCUSSION OF DECOHERENCE CHANNELS}

The summary of measured coherence times along with the extracted device parameters are given in Table I. Using this information, we can place important bounds on various decoherence mechanisms, which are summarized in the table and discussed in this section.

\section{A. Flux noise}

Coherence times measured away from the sweet spot as a function of the flux [Figs. 4(a) and 4(b)] agree with a $1 / f$ flux-noise model [Eq. (A1)] with the amplitude approximately equal to $2 \times 10^{-6}(h / 2 e) / \mathrm{Hz}^{1 / 2}$ at $1 \mathrm{~Hz}$ [Eq. (A2)], which is similar to the noise level in flux qubits [14]. However, here the off-sweet-spot coherence time is $2-3$ orders of magnitude longer (a few microseconds) due to the large number of junctions $N$, and hence, the proportionally reduced first-order flux sensitivity [24], whose maximal value is given by $2 \pi E_{L} \times 2 e / h \propto 1 / N$. Having measured both the transition frequency vs flux and the flux-noise amplitude, we can estimate the limit on the coherence time at the sweet spot due to the second-order coupling [Eq. (A3)]. The coupling can be approximated by $(2 \pi)^{4} E_{L}^{2} / f_{01} \times(2 e / h)^{2}$, which gives a range of coherence times $10-100 \mathrm{~ms}$ for the typical devices presented here [24]. Such long times are possible entirely due to the $1 / N^{2}$ scaling of the second-order flux sensitivity.

Interestingly, the data show no signatures of flux-noiseinduced energy relaxation reported in recent experiments on flux qubits $[15,16]$. Formally, the relaxation rate is given by the familiar Fermi's golden rule expression [38] where the spectral density of the bath is replaced by that of the flux noise, such that it grows at low frequencies [Eq. (A4)]. In the case of a fluxonium, the relaxation rate scales as $E_{L}^{2} \sim 1 / N^{2}$ (the square of the energy matrix element in Fermi's golden rule). This gives protection against the suggested energy relaxation by a factor of $10^{2}-10^{4}$ in comparison to flux qubits. Such a large protection factor may explain the dramatic difference between the $T_{1}$ vs qubit frequency data reported in Refs. $[15,16]$ and that shown in Fig. 3 here.

\section{B. Out-of-equilibrium quasiparticles}

A qubit can relax by emitting a photon which is absorbed by an unpaired quasiparticle tunneling across a junction $[39,40]$. Assuming that the $T_{1}$ values in Table I are limited by the tunneling of out-of-equilibrium quasiparticles across the chain junctions, we conclude that their normalized density $x$ can be below $10^{-8}$ [Eq. (A14)]. Given the device dimensions, this number corresponds to less than one quasiparticle in the entire chain.

According to theory, the tunneling across the weak junction is coherently suppressed at $\phi_{\text {ext }}=\pi$ by the destructive electron-hole interference [41]. The interpretation of a previous fluxonium experiment in terms of this effect [42] implied a 2 orders of magnitude difference between the values of $x$ for the weak junction and for the chain junctions [43], which seems unlikely. In our experiment, an estimate of the quasiparticle density near the small junction can be obtained from device $C$ which has a double loop. There, the coherent suppression of tunneling is absent even at the double sweet spot of both loops because one of the two weak junctions is always away from the $\pi$-phase bias [22]. Yet, the measured relaxation rate is similar to those of the single-loop devices. Its value requires the absence of quasiparticles near the weak junction, consistent with the above conclusion regarding the absence of quasiparticles in the chains. We cautiously speculate that vortices may be efficient at trapping quasiparticles in our specific device geometry [44], although further experimental results are needed to verify this hypothesis.

\section{Out-of-equilibrium photons}

Every qubit undergoing a dispersive readout will experience dephasing due to photon shot noise if the readout mode is not properly thermalized [37]. In this work, we deliberately avoid this issue by making the readout mode linewidth $\kappa$ much larger than the dispersive shift $\chi$ [see Eq. (A15) and Table I]. As Table I illustrates, there is no obvious correlation between $\chi$ and $T_{2}$, which is evidence that thermal photons in the readout mode are indeed irrelevant.

Of more importance is the photon shot noise in the $N-1$ collective modes of the Josephson chain [45]. Their frequencies bunch near the junction's plasma frequency, which in our case is around $20 \mathrm{GHz}$. Because of the nonlinearity of the plasma modes, occupation of one such mode by a single photon introduces a dispersive shift of about $10^{-4}$ of the qubit frequency. Such a shift is much larger than the qubit's natural linewidth [46]. Hence, in order for the qubit to have a coherence time $T_{2}$, the average time for the absence of an out-of-equilibrium photon excitation in each mode must be longer than $N \times T_{2}$. Given the numbers in Table I, we estimate this time to be longer than $50 \mathrm{~ms}$, which means that the chain is practically empty of out-of-equilibrium photons. The thermalization of plasma modes in our chains is intriguing because the microwave environment at such high frequencies is poorly characterized. On one hand, with the external quality factor $Q \approx 500$, our cavity is an order of magnitude more open to the readout line than those typically used in high-coherence transmon experiments. Hence, the circuit modes are well exposed to the environment of the readout line. On the other hand, losses in coaxial components in this line are substantially higher at $20 \mathrm{GHz}$, and this might lead to a better thermalization of the high-frequency modes.

\section{Dielectric loss}

Because our devices have capacitive antennas, they are exposed to surface loss in the same way as any other 
capacitively shunted junction qubit. Small temporal fluctuations of $T_{1}$ may be consistent with the recent data on the $X$-mon qubits explained by the drifts in the value of $\tan \delta_{C}$ due to the dynamics of the weakly coupled two-level defects [47]. Fluctuations aside, the relaxation time $T_{1}$ grows upon reducing the qubit frequency, in agreement with the model of a weakly frequency-dependent dielectric loss tangent $\tan \delta_{C} \propto \omega^{\epsilon}$ of the total capacitance $C$ across the weak junction. Let us first comment on this frequency dependence. Unlike most energy relaxation measurements of superconducting qubits, the data in Fig. 3 span a remarkable six octaves of frequency, which makes it more sensitive to the possible frequency dependence of $\tan \delta_{C}$. We use the values $\epsilon=0.15$ and $T=20 \mathrm{mK}$ (the base temperature of our dilution refrigerator) in Eqs. (A5)-(A10) to match the theory and data in Fig. 3. However, it is likely that the device temperature is higher, and this will require a larger value of $\epsilon$. More accurate experiments are required to establish bounds on the value of $\epsilon$ to clarify the underlying mechanism. Note that having $\epsilon>0$ is a fortunate circumstance because it helps to increase the energy relaxation time toward lower frequencies.

The absolute values of $T_{1}$ at the sweet spot can be explained assuming a narrow range of $\tan \delta_{C} \approx(2.0-3.6) \times$ $10^{-6}$ taken at the frequency of $6 \mathrm{GHz}$ (Fig. 3). Importantly, these numbers are nearly an order of magnitude larger than those extracted for optimally designed 3D transmons [11], where $\tan \delta_{C}$ reaches down to $\tan \delta_{C} \approx 3 \times 10^{-7}$. Given that our antenna has a similar geometry, we expect it to have a similar surface participation ratio [see Eq. (A11)]. Therefore, our relaxation data strongly suggest that losses in the interface layers of our devices are significantly stronger than those of optimally fabricated transmons. This is not unexpected, given that our simplistic $\mathrm{Al}$ on $\mathrm{Si}$ fabrication procedure does not involve any advance surface preparation steps. In fact, the production of device $I$, which corresponds to a notably smaller value of $\tan \delta_{C}$ (Fig. 3, bottom and Table I), includes an extra step of dipping the chip into a buffer oxide etching solution prior to spinning resist (see Appendix Sec. VII). This result is additional evidence of surface loss dominating the energy relaxation.

Dielectric loss in the tunnel oxide of the chain junctions is another potentially important energy relaxation mechanism. Assuming that each junction's capacitance has a nonzero loss tangent, we can estimate its average value as $\tan \delta_{\text {AlOx }}<10^{-3}$. Fortunately, the large number of junctions in the chain helps to reduce the relaxation rate proportionally to $1 / N$. This is because the alternating voltage across the antenna is divided by $N$ for each junction of the chain [24]. Our estimate on the loss tangent of AlOx $\left(10^{-4}\right)$ is about an order of magnitude smaller than the previously reported bulk value [48]. It is possible that the area of each chain junction (about $1 \mu \mathrm{m}^{2}$ ) is sufficiently low to make encountering a strongly coupled charge defect statistically unlikely. This effect can be explored in future devices upon varying the chain junction area.

\section{E. Inductive loss tangent}

By analogy with the effective dielectric loss tangent $\tan \delta_{C}$ of the capacitance $C$, we can introduce an empirical inductive loss tangent $\tan \delta_{L}=\operatorname{Im}[L] / \operatorname{Re}[L]$ for the inductance $L$. One can show that the two loss tangents leading to the same value of $T_{1}$ are related according to $\tan \delta_{L} / \tan \delta_{C}=\omega_{01}^{2} L C$ [see Eq. (A12)]. For a harmonic oscillator, the ratio above is unity, which means that $T_{1}$ is determined by the mechanism with the largest loss tangent. By contrast, our devices are much more sensitive to the absolute value of the inductive loss tangent rather than to an absolute value of the capacitive loss tangent because for our parameters $\omega_{01}^{2} L C \sim 10^{-1}-10^{-2}$. Given that for the quasiparticles loss $\tan \delta_{L} \approx x$, one can qualitatively understand the nearly 2 orders of magnitude difference between the bounds on $\tan \delta_{C}$ and $x$ in Table I.

We conclude that $T_{2}$ is largely limited by $T_{1}$, and the most likely explanation for the measured $T_{1}$ times in all devices is the surface loss in the antenna. By fabricating our fluxoniums either on sapphire $\left(\mathrm{Al}_{2} \mathrm{O}_{3}\right)$ or on a properly surface-treated silicon, relaxation time $T_{1}$ is expected to grow. A modest improvement in $\tan \delta_{C}$ by a factor of $2-3$ is sufficient to explore the limits to coherence at the level of $1 \mathrm{~ms}$.

\section{TOWARD QUANTUM COMPUTING WITH FLUXONIUMS}

How can high-coherence fluxonium qubits interact strongly on demand and undergo fast two-qubit gates? This question is especially relevant because fluxoniums achieve their superior coherence largely due to the drastic reduction of the qubit transition frequency. Here we outline how fluxoniums can be integrated into existing schemes of scalable quantum computing. This outlook discussion is largely based on already published theoretical and experimental work on interacting fluxoniums.

\section{A. Capacitive coupling}

Viewing fluxoniums as inductively shunted charge qubits, one can understand the effect of connecting two such devices by a mutual capacitance $C_{C}$ using the charge qubit expressions. Assuming that $C_{C} \ll C$, the effective interaction term is given by $H_{\text {int }}=J_{C} n_{1} n_{2}$, where $J_{C}=2 E_{C} \times\left(C_{C} / C\right)$, and $n_{1,2}$ are the charge operators of the two devices. Given that in our design $E_{C} \sim 1 \mathrm{GHz}$, and choosing a moderate ratio $C_{C} / C=0.1$, one can readily get an exchange constant $J_{C} \sim 200 \mathrm{MHz}$, which is similar to what is possible with capacitive coupling of transmons. Note that the repulsion of qubit frequencies by the term $H_{\text {int }}$ is significantly reduced by the small value of the charge matrix elements $\left\langle 0\left|n_{1,2}\right| 1\right\rangle$. Fortunately, this is not the case 
for transitions outside the computational subspace, where matrix elements are large, and one can use them to perform, e.g., microwave-activated two-qubit gates [26].

\section{B. Inductive coupling}

In contrast with capacitive coupling, a proper inductive coupling of fluxoniums allows for extremely strong interactions already within the computational subspace. Viewing fluxoniums as superconducting loops with a weak link, two such devices can be inductively coupled by sharing one or several junctions between the two loops. Assuming that the fraction of the shared junctions is $m \ll 1$, the interaction term is given by $H_{\text {int }}=-J_{L}\left(\phi_{1} / \pi\right)\left(\phi_{2} / \pi\right)$, where $J_{L}=m \pi^{2} E_{L}$ and $\phi_{1,2}$ are the phase operators of the two devices. Normalization of the phase operators by $\pi$ is convenient because $\left\langle 0\left|\phi_{1,2}\right| 1\right\rangle \approx \pi$ at the sweet spot. Therefore, even for a modest $m=0.1$ (about 10-20 shared junctions), we get the frequency repulsion of qubit transitions on the order of $J_{L} \approx 0.5 \mathrm{GHz}$; i.e., it is comparable to transition frequencies. In fact, by making $m \sim 1$, a molecular-type binding of two fluxoniums has already been experimentally demonstrated [27].

\section{Dispersive qubit-cavity interaction}

Circuit quantum electrodynamics with fluxonium qubits was described in Ref. [31]. The most useful strong dispersive regime of circuit QED corresponds to $\chi \gg \kappa$. Introducing the dimensionless photon creation (annihilation) operator $a^{\dagger}(a)$, the coupling term is $H_{\text {int }}=g_{C} n i\left(a-a^{\dagger}\right)$ for capacitive coupling and $H_{\text {int }}=g_{L} \phi\left(a+a^{\dagger}\right)$ for inductive coupling. Given the discussion of inductive and capacitive coupling above, it is straightforward to achieve the values $g_{C} / 2 \pi, g_{L} / 2 \pi \approx 100 \mathrm{MHz}$, typical of conventional qubits $[22,29]$. However, here the qubit frequency (typically $500 \mathrm{MHz}$ ) is far detuned from the photon frequency (typically above $5 \mathrm{GHz}$ ). Nevertheless, the shifts $\chi$ can be large due to the virtual transitions connecting states $|0\rangle$ and $|1\rangle$ to the noncomputational states [25]. In fact, it was shown experimentally [22] that dispersive shift is nonzero even for a vanishing qubit transition dipole, i.e., $\langle 0|\phi, n| 1\rangle \rightarrow 0$.

\section{Flux-controlled gates}

Since fluxonium's spectrum can be tuned by flux, it is tempting to consider flux-controlled gate operations. Perhaps the simplest such gate is the analog of a $C$-phase gate for transmons [49] relying on the repulsion of the twoqubit states $|11\rangle$ and $|20\rangle$ or $|02\rangle$. The states repulsion can be generated by a direct capacitive or inductive connection of fluxoniums. In fact, one may expect an enhancement of gate fidelity because fluxoniums maintain a relatively highcoherence time $T_{2} \approx 5 \mu$ s while biased outside the sweet spot during the gate operation.

\section{E. Fixed-frequency qubit gates}

A recent proposal described a fast $\mathrm{CZ}$ gate between two capacitively or inductively coupled fluxoniums obtained by applying a $\pi$ pulse to the transition 1-2 of the target qubit, whose frequency shifts depending on the state of the control qubit [26]. In general, the quantum-state leakage during such gate operations shows to be remarkably low owing to the large anharmonicity of the noncomputational part of the spectrum. Although microwave-activated gates still require static flux biasing of qubits at their sweet spots, their advantage is that they are compatible with the $3 \mathrm{D}$ circuit QED architecture used in this work.

\section{F. Quantum adiabatic optimization}

A network of interconnected fluxoniums after the projection to the computational subspace can implement a generic quantum spin-1/2 Hamiltonian:

$$
H=\sum_{i, j} h_{i}^{Z} \sigma_{Z_{i}}+h_{i}^{X} \sigma_{X_{i}}-J_{i, j}^{X X} \sigma_{X_{i}} \sigma_{X_{j}}
$$

Here, the field $h_{Z}$ is the qubit transition frequency at the sweet spot, field $h_{X}$ is the detuning from the sweet spot, and $J_{X X}$ is the nearest-neighbor coupling constant. The field $h_{Z}$ can also be tuned independently from $h_{X}$ by replacing a single weak junction by a split junction, as is done in device $C$. Such a Hamiltonian is typically implemented using the system of semiclassical superconducting quantum-interference-device circuits to explore quantumannealing algorithms [50].

Our devices can provide a previously unavailable realization of this model. (i) Owing to the extremely large anharmonicity $\omega_{12} / \omega_{01} \gg 1$, a network of fluxoniums remains in its computational subspace even in the presence of multiple spin flips; i.e., projecting to the computational subspace remains valid. (ii) Even far away from the sweet spot, we get $T_{2} \sim 5 \mu \mathrm{s}$, which translates into the level broadening of about $30 \mathrm{kHz}$. This number, in principle, allows us to resolve the many-body level spacing in a system of ten locally coupled spins. (iii) Inductive connection allows a local coupling to multiple neighbors with the condition $J_{X X} \sim h_{Z}$. These three conditions are simultaneously required for exploring the most intriguing scenarios of quantum many-body physics of spin systems. Fluxoniums are therefore well positioned for constructing the next generation of quantum annealers operating in a highly coherent regime where quantum speed up is expected from theory [4].

\section{G. Optimal qubit frequency}

It is interesting to discuss the choice of the optimal qubit frequency, as the design, in principle, allows us to reduce it to an arbitrary low value. We believe that the presented 
qubit frequency range around $500 \mathrm{MHz}$ is currently the optimal for a number of reasons.

The first concern is the finite temperature of the qubit. Already at $500 \mathrm{MHz}$, which translates to a temperature of $25 \mathrm{mK}$, a significant population of state $|1\rangle$ is expected. In principle, this frequency is not a problem for a quantum processor as long as the energy relaxation time $T_{1}$ is sufficiently long. The qubits anyway need to be initialized with a high fidelity. However, it is convenient to be able to characterize low-frequency devices without the need to do so; hence, keeping the qubit frequency not far below the temperature is advantageous. More importantly, for $\hbar \omega \ll k_{B} T$, the relaxation time $T_{1}$ must be rescaled compared to its zero-temperature value due to the stimulated emission factor $T_{1} \approx T_{1}(T=0) \times\left(k_{B} T / \hbar \omega\right)$.

From a technical viewpoint, the chosen frequency range appears particularly convenient for scaling: There is room to frequency resolve neighboring qubits by spreading them by a few hundred MHz; Rabi driving with a frequency up to $50 \mathrm{MHz}$ can be applied even within the 3D circuit QED architectures, which can provide 10-ns-long single-qubit pulses; cross talks are in general expected to be reduced at lower frequencies. Last but not least, qubit pulses can be done using cheaper digital electronics which can significantly reduce scaling costs.

\section{SUMMARY}

We present a specific design of fluxonium qubits which repeatedly yield high-coherence times $T_{2}$ at the half-integer flux bias, with the best device satisfying $T_{2}>400 \mu \mathrm{s}$. To our knowledge, this is the longest coherence time found in a superconducting qubit today. Compared to a typical flux qubit, the effect of $1 / f$ flux noise is practically eliminated by the large loop inductance $\left(L \sim 10^{2} \mathrm{nH}\right)$ of the Josephson chain. The qubit transition frequency can still be flux tuned by many octaves while keeping the coherence time above a few microseconds, limited by first-order coupling to flux noise. Our fluxoniums are compatible with transmon-based scaling architectures, which require connecting an external coupling capacitance (antenna) to the small junction. This connection comes at the price of energy relaxation induced by the surface loss in the antenna. The surface loss problem is largely (but not completely) bypassed by reducing the qubit frequency to around $\omega_{01} / 2 \pi \approx 500 \mathrm{MHz}$. Because the spectral density of noise associated with surface loss drops rapidly with frequency, the relaxation time of our qubits exceeds that of the best capacitively shunted junction circuits despite a suboptimal $\mathrm{Al}$ on $\mathrm{Si}$ fabrication procedure.

Several design features possibly contribute to the dramatic improvement of coherence compared to previous fluxonium experiments. The original design was severely limited by the surface loss $\left(T_{2} \sim T_{1} \lesssim 10 \mu \mathrm{s}\right)$ in the finger capacitors attached to the small junction [21,24]. In a subsequent series of experiments, this capacitance was removed, and the readout was done through the inductive coupling to a low quality-factor chain mode dressed by an external capacitance $[27,29,42,43]$. This approach expectedly eliminated the surface loss and dramatically improved $T_{1}$ but not $T_{2}$. In the present work, we go back to the original design $[21,24]$, including the Dolan bridge fabrication and (i) use a better geometry of the antenna to minimize the surface participation ratio the way it is done in modern transmons [11] and (ii) use the fundamental mode of a simple copper box for readout [51]. Finally, the geometry of our Josephson chain might be favorable for trapping quasiparticles in the background magnetic field.

Importantly, the approximately tenfold reduction of the qubit frequency compared to the traditional $5-\mathrm{GHz}$ value does not prevent fast gate operations or strong interactions. This is because the spectrum of a typical fluxonium has a rich structure of excited states reminiscent of the fine structure in the spectrum of conventional atoms. Transitions outside the computational subspace belong to a traditional frequency and transition dipole range, which, as we outline in Sec. V, can be utilized for creating fast flux- or microwave-activated quantum gates. Moreover, the inductive connection of fluxoniums via shared junctions can make the exchange coupling comparable to the qubit frequency without leaving the computational subspace. These large exchange couplings combined with the exceptional coherence times can be especially useful for constructing coherent quantum annealers. The next steps toward quantum computing with fluxoniums consist of demonstrating high coherence in combination with fast single-shot readout, fast flux tuning, and fast two-qubit gates.

In closing, let us remark on our specific Josephson chain implementation of large loop inductances required by fluxoniums. Following the common viewpoint of minimizing the number of junctions per qubit, one may be tempted to replace the discrete chain by a patterned film of a highly disordered superconductor with a comparable kinetic inductance. It is important to realize that the effective loss tangent of the inductance must be in the $10^{-7}-10^{-8}$ range in order to reach the coherence times reported in this experiment. Whether such a low loss can be reached with dirty superconductors is an interesting question [52-56]. Our specific Josephson tunnel junction chain design [Fig. 1(c)] is primarily motivated by the maximal simplicity of the qubit fabrication procedure: It is single step and does not require high-contrast lithography. The high coherence is made possible, in part, by the remarkably good thermalization of both quasiparticles and collective modes in the chains. Understanding this effect in future experiments may have high impact on quantum-circuit design. In the meantime, our experiment demonstrates for the first time that the coherence time of superconducting qubits can be extended beyond the state of the art by increasing circuit complexity.

\section{ACKNOWLEDGMENTS}

We like to acknowledge fruitful conversations with Maxim Vavilov, Chen Wang, Benjamin Huard, Ivan 
Pechenezhskiy, and Konstantin Nesterov. This work was supported by Sloan Research Fellowship, by National Science Foundation via Career Award (DMR 1455261) and Physical Frontier Center at the Join Quantum Institute (No. 1430094), and by ARO-LPS HiPS program (No. W911NF-18-1-0146).

\section{APPENDIX}

\section{Circuit QED}

The reported fluxonium devices are mounted inside copper cavities and measured dispersively. The cavities are designed to have the fundamental resonant mode at approximately $7.5 \mathrm{GHz}$. For devices $A-F$ the qubit-cavity vacuum coupling coefficient $g / 2 \pi \approx 70 \mathrm{MHz}$. Devices $G-I$ have smaller antennas, resulting in a smaller coupling to the cavity, $g / 2 \pi \approx 40 \mathrm{MHz}$. Devices $A-H$ are mounted on copper cavities with overcoupled output port, $\kappa / 2 \pi \approx$ $15 \mathrm{MHz}$. Device $I$ is measured inside a cavity with $\kappa / 2 \pi \approx 4 \mathrm{MHz}$.

\section{Flux noise}

An inherent decoherence source in solid-state devices is $1 / f$ flux noise found to originate from surface defects on the substrate. Its associated spectral density

$$
S_{\Phi}(\omega)=2 \pi \frac{A^{2}}{\omega}
$$

is found to affect superconducting flux qubits across more than an order of magnitude in frequency [15]. Measurements utilizing flux-sensitive devices find the noise amplitude to vary between $10^{-5}$ and $10^{-6} \Phi_{o}$ [15].

Away from the sweet spot, the qubit is sensitive to firstorder flux noise, and it dephases following a Gaussian function with rate

$$
\Gamma_{\Phi}^{\text {Echo }}=\frac{\partial \omega}{\partial \Phi} A \sqrt{\ln 2} .
$$

Measurement of $T_{2}^{\text {Echo }}$ away from sweet spot gives us the noise amplitude $A \approx 1.8 \mu \Phi_{o}$. The large shunting inductance lowers the qubit's first-order sensitivity, and we are able to achieve a few microseconds decoherence time despite the flux-noise amplitude being similar to the values reported previously. As the flux is tuned to a half-integer flux, the qubit's first-order flux sensitivity goes to zero. However, the second-order sensitivity $\partial^{2} \omega / \partial \Phi^{2}$ reaches a local maxima, giving rise to the concern that flux noise can still limit a qubit's coherence. The relevant dephasing rate is [57]

$$
\Gamma_{\Phi^{2}}=\frac{\partial^{2} \omega}{\partial \Phi^{2}} A^{2} .
$$

Because of the low-flux-noise amplitude in our system, the second-order effect is small, resulting in an upper $T_{\phi}$ limit $>10 \mathrm{~ms}$ for all qubits.
Another potential detriment coming from flux noise at half-integer fluxes is energy relaxation. The noise will couple to the qubit via the persistent current $\hat{I}_{p}=\hat{\Phi} / L$, resulting in a relaxation rate [16]

$$
\Gamma_{i j}^{\Phi}=\frac{1}{(2 e)^{2}} \frac{1}{L^{2}}|\langle j|\hat{\phi}| i\rangle|^{2} S_{\Phi}\left(\omega_{i j}\right),
$$

where $\phi=(2 e / \hbar) \Phi$. Again, the large inductance of the chain plays an important role: The relaxation time $T_{1}$ due to flux noise increases with $L^{2}$. Thus, it is in the order of hundreds of milliseconds and is not a concern in fluxonium qubits. This result is quite remarkable compared to typical flux-tunable qubits where $T_{1}$ 's are typically limited by flux noise at the low-frequency sweet spot.

\section{Dielectric loss}

Dielectric loss in fluxonium can be modeled as a lossy shunting capacitor with admittance $Y_{C}(\omega)$. Using Fermi's golden rule [58], we can write the associated relaxation rate as

$$
1 / T_{1}(\omega)=\frac{1}{(2 e)^{2}}|\langle 0|\hat{\phi}| 1\rangle|^{2} S_{\text {diel }}(\omega),
$$

where the spectral density of the noise of a lossy capacitance at zero temperature $(T=0)$ coupled to the phase $\phi$ across the weak junction is given by

$$
S_{\text {diel }}(\omega)=2 \hbar \omega \operatorname{Re}\left[Y_{C}(\omega)\right]
$$

and

$$
\operatorname{Re}\left[Y_{C}(\omega)\right]=\omega C / Q_{\text {diel }}(\omega) .
$$

Here, $C$ is the effective capacitance across the weak junction experimentally determined from $E_{C}=e^{2} / 2 C$, and the dielectric quality factor $Q_{\text {diel }}$ is usually defined using the effective loss tangent of the capacitance $C$ according to $Q_{\text {diel }}=1 / \tan \delta_{C}$. In general, this quantity can depend on the frequency, which we model in this paper using a phenomenological power law

$$
\frac{\tan \delta_{C}(\omega)}{\tan \delta_{C}(\omega=2 \pi \times 6 \mathrm{GHz})}=\left(\frac{\omega}{2 \pi \times 6 \mathrm{GHz}}\right)^{\epsilon} .
$$

At a nonzero temperature, the expression for $S_{\text {diel }}$ is modified due to the stimulated emission by thermal photons in the lossy environment according to

$$
\left.S_{\text {diel }}(\omega) \rightarrow S_{\text {diel }}\right|_{T=0} \times\left[\operatorname{coth}\left(\hbar \omega / 2 k_{B} T\right)+1\right] / 2 .
$$

Moreover, the energy relaxation rate, which we still call $T_{1}$ for simplicity, is the sum of rates to transition up and down. Therefore, to compare the measured relaxation times to 
theory at a finite temperature, we must modify the expression for $T_{1}$ according to

$$
1 / T_{1} \rightarrow 1 / T_{1}(\omega)+1 / T_{1}(-\omega) .
$$

Thus, in order to produce the dashed theory lines shown in Fig. 3, we use Eqs. (A10) and (A9) with $T=20 \mathrm{mK}$, $\epsilon=0.15$, and $\tan \delta_{C}=(2-3.6) \times 10^{-6}$.

Finally, let us note that the value of the effective loss tangent $\tan \delta_{C}$ can be understood as a contribution from losses in various parts of the circuit characterized by the materials loss tangents $\delta_{i}, i=1,2, \ldots$ according to

$$
\tan \delta_{C}=\sum_{i} p_{i} \tan \delta_{i}
$$

The participation factors $p_{i}$ are usually determined by the circuit geometry. For example, oxide between metal-substrate, metal-air, and substrate-air interfaces dominate over the bulk loss of the substrate material. Our antennas have a similar geometry to those of the 3D transmons, and hence, we expect a similar participation ratio. Therefore, in order to compare the quality of the surfaces and interfaces, it is sufficient to compare the values of $\tan \delta_{C}$, which follow directly from $T_{1}$ measurement.

\section{Inductive loss}

Analogous to dielectric loss, we can prescribe a lossy inductor $L \rightarrow L\left(1+i \tan \delta_{L}\right)$ and write the associated admittance as

$$
\operatorname{Re}\left[Y_{L}(\omega)\right]=\frac{\tan \delta_{L}}{\omega L} .
$$

For the same relaxation time $T_{1}$, the ratio between the inductive loss tangent and dielectric loss tangent is

$$
\frac{\tan \delta_{L}}{\tan \delta_{C}}=\frac{(\hbar \omega)^{2}}{8 E_{C} E_{L}},
$$

which is much smaller than unity. This implies that our typical inductor (chain) is relatively free from dissipative impurities.

\section{Quasiparticles}

Nonequilibrium quasiparticles have been shown to limit a qubit's energy relaxation time $T_{1}$ and coherence time $T_{2}$. At the $\phi_{\text {ext }}=\pi$ sweet spot in fluxonium, quasiparticle tunneling effect across the small junction is suppressed [42]. However, if there are quasiparticles in the chain, they still cause energy relaxation $[41,43]$ at the rate

$$
\Gamma_{1}\left(\omega_{01}\right)=\left|\left\langle 0\left|\frac{\hat{\varphi}}{2}\right| 1\right\rangle\right|^{2} \frac{8 E_{L}}{\pi \hbar} x_{\mathrm{QP}} \sqrt{\frac{2 \Delta}{\hbar \omega_{01}}} .
$$

Our measured $T_{1}$ indicates that $x_{\mathrm{QP}}$ is on the order of $10^{-8}$ corresponding to less than a single quasiparticle in the entire chain. We emphasize that this is the high limit for the number of quasiparticles in the chain, as we have yet observed any effect from them.

\section{Cavity temperature}

The dephasing rate due to residual cavity photons $n_{\mathrm{th}}$ in the low photon number follows [27]

$$
\Gamma_{\phi}^{\mathrm{th}}=\frac{\bar{n}_{\mathrm{th}} \kappa \chi^{2}}{\kappa^{2}+\chi^{2}}
$$

In our experiments, due to the small $\chi / \kappa$ ratio, the number of cavity thermal photons previously reported in other labs [15] corresponds to a very high $T_{\phi}^{\text {th }}$ limit.

\section{Step-by-step fabrication procedure}

We follow the procedure below to fabricate the fluxonium qubits reported in this paper. Chip cleaving: First, we apply isopropyl alcohol (IPA) on the wafer and blow dry with $\mathrm{N}_{2}$. Then, we scribe the wafer with a diamond-tip pen, rinse it with IPA, and blow dry again. Finally, we cleave the wafer into $9 \mathrm{~mm} \times 4 \mathrm{~mm}$ chips. Chip cleaning: We sonicate the chip in acetone for $3 \mathrm{~min}$, repeat sonication with IPA, then blow dry with $\mathrm{N}_{2}$. As an exception, device $I$ is cleaned using a buffer oxide etch, and then the cleaning steps described above are applied to it. Resist application: For the first resist layer, we spin MMA EL 13 at $5000 \mathrm{rpm}$ for $1 \mathrm{~min}$, then bake the chip at $180^{\circ} \mathrm{C}$ for $1 \mathrm{~min}$. For the second resist layer, we spin 950 PMMA A3 at $4000 \mathrm{rpm}$ for $1 \mathrm{~min}$, then bake the chip at $180^{\circ} \mathrm{C}$ for $30 \mathrm{~min}$. We inspect the chip after each spin and before electron-beam lithography for defects. Electron-beam writing: We use a 100-kV ELS Elionix with 1-nA beam current to write the lithography pattern on the chip. As an exception, the $C$ device is fabricated using a $20-\mathrm{kV}$ converted scanning electron microscope. Development: We place the chip in a 3 IPA:1 DI solution at $6{ }^{\circ} \mathrm{C}$ and gently shake it for $2 \mathrm{~min}$. Deposition: We load the chip into the Plassys evaporator and pump the loadlock for $20 \mathrm{~h}$ before the deposition. We check that the loadlock pressure reaches $1.3 \times$ $10^{-7}$ mbar before beginning deposition. The deposition steps include the following:

(i) 20-s descum with argon at each deposition angle $\left( \pm 23.83^{\circ}\right)$

(ii) Titanium deposition at a rate of $0.1 \mathrm{~nm} / \mathrm{s}$ for $2 \mathrm{~min}$.

(iii) First $\mathrm{Al}$ deposition at a rate of $1 \mathrm{~nm} / \mathrm{s}$ at an angle of $23.83^{\circ}$, resulting in approximately $20 \mathrm{~nm}$ of $\mathrm{Al}$

(iv) 10 -min oxidation at 100 mbar

(v) Second $\mathrm{Al}$ deposition at a rate of $1 \mathrm{~nm} / \mathrm{s}$ at $-23.83^{\circ}$; approximately $40 \mathrm{~nm}$ of $\mathrm{Al}$ is deposited

(vi) 20-min oxidation at 10 mbar

Liftoff: We place the chip in acetone and put the beaker on a hot plate at $60^{\circ} \mathrm{C}$ for $3 \mathrm{~h}$. We then sonicate it for $5 \mathrm{~s}$ and 
sonicate again in IPA for $10 \mathrm{~s}$. Finally, we blow dry the chip with $\mathrm{N}_{2}$.

\section{Low-temperature measurement setup}

We utilize a minimal setup [Fig. 5] to perform standard microwave transmission measurements at low temperatures. The setup [Fig. 5] is installed in a BlueFors LD-250 dilution refrigerator and consists of mostly commercially available microwave components. The input coaxial line contains 20-dB attenuators from XMA at 3-K, 100-mK, and $20-\mathrm{mK}$ stages of the dilution refrigerator. In addition, we use a low-pass filter at $20 \mathrm{mK}$ from K\&L with a cutoff at $12 \mathrm{GHz}$. The output line contains three Quinnstar isolators in the (4-8)-GHz band and a K\&L filter between the isolators and the low-temperature HEMT amplifier. Eccosorb CR110 low-pass filters are placed at the 3-K stage below the 20-dB attenuator on the input line and the HEMT amplifier on the output line. Note that the effect of these filters remains inconclusive so far. The space below the $20-\mathrm{mK}$ stage is protected by a gold-plated radiation shield. The sample is mounted inside a copper box resonator using a small amount of pressed-indium contacts. The copper cavity is then mounted on a cold finger inside a single cylindrical cryogenic cryoperm shield.

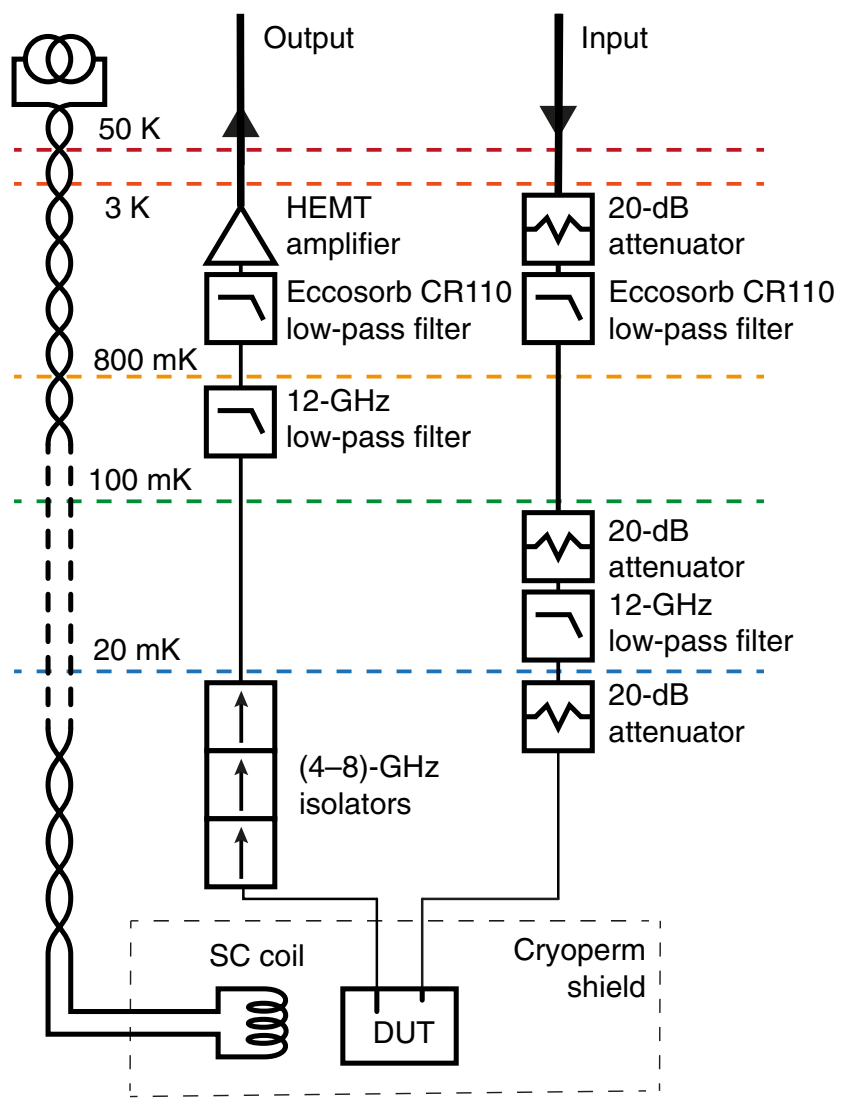

FIG. 5. Cryogenic experimental setup.
[1] M.H. Devoret and R.J. Schoelkopf, Superconducting Circuits for Quantum Information: An Outlook, Science 339, 1169 (2013).

[2] R. J. Schoelkopf and S. M. Girvin, Wiring Up Quantum Systems, Nature (London) 451, 664 (2008).

[3] R. Barends, J. Kelly, A. Megrant, A. Veitia, D. Sank, E. Jeffrey, T. C. White, J. Mutus, A. G. Fowler, B. Campbell et al., Superconducting Quantum Circuits at the Surface Code Threshold for Fault Tolerance, Nature (London) 508, 500 (2014).

[4] S. Boixo, V. N. Smelyanskiy, A. Shabani, S. V. Isakov, M. Dykman, V. S. Denchev, M. H. Amin, A. Yu. Smirnov, M. Mohseni, and H. Neven, Computational Multiqubit Tunnelling in Programmable Quantum Annealers, Nat. Commun. 7, 10327 (2016).

[5] A. Kandala, A. Mezzacapo, K. Temme, M. Takita, M. Brink, J. M. Chow, and J. M. Gambetta, Hardware-Efficient Variational Quantum Eigensolver for Small Molecules and Quantum Magnets, Nature (London) 549, 242 (2017).

[6] R. Versluis, S. Poletto, N. Khammassi, B. Tarasinski, N. Haider, D. J. Michalak, A. Bruno, K. Bertels, and L. DiCarlo, Scalable Quantum Circuit and Control for a Superconducting Surface Code, Phys. Rev. Applied 8, 034021 (2017).

[7] C. Song, K. Xu, W. Liu, C.-P. Yang, S.-B. Zheng, H. Deng, Q. Xie, K. Huang, Q. Guo, L. Zhang, P. Zhang, D. Xu, D. Zheng, X. Zhu, H. Wang, Y.-A. Chen, C.-Y. Lu, S. Han, and J.-W. Pan, 10-Qubit Entanglement and Parallel Logic Operations with a Superconducting Circuit, Phys. Rev. Lett. 119, 180511 (2017).

[8] J. S. Otterbach, R. Manenti, N. Alidoust, A. Bestwick, M. Block, B. Bloom, S. Caldwell, N. Didier, E. S. Fried, S. Hong et al., Unsupervised Machine Learning on a Hybrid Quantum Computer, arXiv:1712.05771.

[9] C. Neill, P. Roushan, K. Kechedzhi, S. Boixo, S. V. Isakov, V. Smelyanskiy, A. Megrant, B. Chiaro, A. Dunsworth, K. Arya et al., A Blueprint for Demonstrating Quantum Supremacy with Superconducting Qubits, Science 360, 195 (2018).

[10] J. M. Martinis, K. B. Cooper, R. McDermott, M. Steffen, M. Ansmann, K. D. Osborn, K. Cicak, S. Oh, D. P. Pappas, R. W. Simmonds et al., Decoherence in Josephson Qubits from Dielectric Loss, Phys. Rev. Lett. 95, 210503 (2005).

[11] C. Wang, C. Axline, Y. Y. Gao, T. Brecht, Y. Chu, L. Frunzio, M. H. Devoret, and R. J. Schoelkopf, Surface Participation and Dielectric Loss in Superconducting Qubits, Appl. Phys. Lett. 107, 162601 (2015).

[12] R. H. Koch, D. P. DiVincenzo, and J. Clarke, Model for $1 / f$ Flux Noise in Squids and Qubits, Phys. Rev. Lett. 98, 267003 (2007).

[13] F. Yoshihara, K. Harrabi, A. O. Niskanen, Y. Nakamura, and J.S. Tsai, Decoherence of Flux Qubits due to $1 / f$ Flux Noise, Phys. Rev. Lett. 97, 167001 (2006).

[14] J.-L. Orgiazzi, C. Deng, D. Layden, R. Marchildon, F. Kitapli, F. Shen, M. Bal, F. R. Ong, and A. Lupascu, Flux Qubits in a Planar Circuit Quantum Electrodynamics Architecture: Quantum Control and Decoherence, Phys. Rev. B 93, 104518 (2016).

[15] F. Yan, S. Gustavsson, A. Kamal, J. Birenbaum, A. P. Sears, D. Hover, T. J. Gudmundsen, D. Rosenberg, G. Samach, 
S. Weber et al., The Flux Qubit Revisited to Enhance Coherence and Reproducibility, Nat. Commun. 7, 12964 (2016).

[16] C. M. Quintana, Yu Chen, D. Sank, A. G. Petukhov, T. C. White, D. Kafri, B. Chiaro, A. Megrant, R. Barends, B. Campbell et al., Observation of Classical-Quantum Crossover of $1 / f$ Flux Noise and Its Paramagnetic Temperature Dependence, Phys. Rev. Lett. 118, 057702 (2017).

[17] J. S. Kline, M. R. Vissers, F. C. S. da Silva, D. S. Wisbey, M. Weides, T. J. Weir, B. Turek, D. A. Braje, W. D. Oliver, Y. Shalibo et al., Sub-Micrometer Epitaxial Josephson Junctions for Quantum Circuits, Supercond. Sci. Technol. 25, 025005 (2012).

[18] P. Kumar, S. Sendelbach, M. A. Beck, J. W. Freeland, Z. Wang, H. Wang, C. C. Yu, R. Q. Wu, D. P. Pappas, and R. McDermott, Origin and Reduction of $1 / f$ Magnetic Flux Noise in Superconducting Devices, Phys. Rev. Applied 6, 041001 (2016).

[19] J. Koch, T. M. Yu, J. Gambetta, A. A. Houck, D. I. Schuster, J. Majer, A. Blais, M. H. Devoret, S. M. Girvin, and R. J. Schoelkopf, Charge-Insensitive Qubit Design Derived from the Cooper Pair Box, Phys. Rev. A 76, 042319 (2007).

[20] M. Steffen, S. Kumar, D. P. DiVincenzo, J. R. Rozen, G. A. Keefe, M. B. Rothwell, and M. B. Ketchen, HighCoherence Hybrid Superconducting Qubit, Phys. Rev. Lett. 105, 100502 (2010).

[21] V.E. Manucharyan, J. Koch, L. I. Glazman, and M. H. Devoret, Fluxonium: Single Cooper-Pair Circuit Free of Charge Offsets, Science 326, 113 (2009).

[22] Y.-H. Lin, L. B. Nguyen, N. Grabon, J. S. Miguel, N. Pankratova, and V.E. Manucharyan, Demonstration of Protection of a Superconducting Qubit from Energy Decay, Phys. Rev. Lett. 120, 150503 (2018).

[23] J. Koch, V. Manucharyan, M. H. Devoret, and L. I. Glazman, Charging Effects in the Inductively Shunted Josephson Junction, Phys. Rev. Lett. 103, 217004 (2009).

[24] V. E. Manucharyan, N. A. Masluk, A. Kamal, J. Koch, L. I. Glazman, and M.H. Devoret, Evidence for Coherent Quantum Phase Slips across a Josephson Junction Array, Phys. Rev. B 85, 024521 (2012).

[25] G. Zhu, D. G. Ferguson, V. E. Manucharyan, and J. Koch, Circuit QED with Fluxonium Qubits: Theory of the Dispersive Regime, Phys. Rev. B 87, 024510 (2013).

[26] K. N. Nesterov, I. V. Pechenezhskiy, C. Wang, V. E. Manucharyan, and M.G. Vavilov, Microwave-Activated Controlled-z Gate for Fixed-Frequency Fluxonium Qubits, Phys. Rev. A 98, 030301(R) (2018).

[27] A. Kou, W. C. Smith, U. Vool, R. T. Brierley, H. Meier, L. Frunzio, S. M. Girvin, L. I. Glazman, and M. H. Devoret, Fluxonium-Based Artificial Molecule with a Tunable Magnetic Moment, Phys. Rev. X 7, 031037 (2017).

[28] I. Chiorescu, Y. Nakamura, C. J. P. M. Harmans, and J. E. Mooij, Coherent Quantum Dynamics of a Superconducting Flux Qubit, Science 299, 1869 (2003).

[29] A. Kou, W. C. Smith, U. Vool, I. M. Pop, K. M. Sliwa, M. Hatridge, L. Frunzio, and M. H. Devoret, Simultaneous Monitoring of Fluxonium Qubits in a Waveguide, Phys. Rev. Applied 9, 064022 (2018).

[30] S. Gladchenko, D. Olaya, E. Dupont-Ferrier, B. Doucot, L. B. Ioffe, and M.E. Gershenson, Superconducting
Nanocircuits for Topologically Protected Qubits, Nat. Phys. 5, 48 (2009).

[31] M. T. Bell, I. A. Sadovskyy, L. B. Ioffe, A. Yu. Kitaev, and M.E. Gershenson, Quantum Superinductor with Tunable Nonlinearity, Phys. Rev. Lett. 109, 137003 (2012).

[32] M. T. Bell, J. Paramanandam, L. B. Ioffe, and M. E. Gershenson, Protected Josephson Rhombus Chains, Phys. Rev. Lett. 112, 167001 (2014).

[33] G. J. Dolan, Offset Masks for Lift-Off Photoprocessing, Appl. Phys. Lett. 31, 337 (1977).

[34] L. Frunzio, A. Wallraff, D. Schuster, J. Majer, and R. Schoelkopf, Fabrication and Characterization of Superconducting Circuit QED Devices for Quantum Computation, IEEE Trans. Appl. Supercond. 15, 860 (2005).

[35] R. Kuzmin, R. Mencia, N. Grabon, N. Mehta, Y.-H. Lin, and V. E. Manucharyan, Quantum electrodynamics of a superconductor-insulator phase transition, Nat. Phys. 15, 930 (2019).

[36] R. Kuzmin, N. Mehta, N. Grabon, R. Mencia, and V.E. Manucharyan, Superstrong Coupling in Circuit Quantum Electrodynamics, arXiv:1809.10739.

[37] D. I. Schuster, A. Wallraff, A. Blais, L. Frunzio, R.-S. Huang, J. Majer, S. M. Girvin, and R. J. Schoelkopf, ac Stark Shift and Dephasing of a Superconducting Qubit Strongly Coupled to a Cavity Field, Phys. Rev. Lett. 94, 123602 (2005).

[38] A. A. Clerk, M. H. Devoret, S. M. Girvin, F. Marquardt, and R. J. Schoelkopf, Introduction to Quantum Noise, Measurement, and Amplification, Rev. Mod. Phys. 82, 1155 (2010).

[39] J. M. Martinis, M. Ansmann, and J. Aumentado, Energy Decay in Superconducting Josephson-Junction Qubits from Nonequilibrium Quasiparticle Excitations, Phys. Rev. Lett. 103, 097002 (2009).

[40] G. Catelani, R. J. Schoelkopf, M. H. Devoret, and L. I. Glazman, Relaxation and Frequency Shifts Induced by Quasiparticles in Superconducting Qubits, Phys. Rev. B 84, 064517 (2011).

[41] G. Catelani, J. Koch, L. Frunzio, R. J. Schoelkopf, M. H. Devoret, and L. I. Glazman, Quasiparticle Relaxation of Superconducting Qubits in the Presence of Flux, Phys. Rev. Lett. 106, 077002 (2011).

[42] I. M. Pop, K. Geerlings, G. Catelani, R J. Schoelkopf, L. I. Glazman, and M.H. Devoret, Coherent Suppression of Electromagnetic Dissipation due to Superconducting Quasiparticles, Nature (London) 508, 369 (2014).

[43] U. Vool, I. M. Pop, K. Sliwa, B. Abdo, C. Wang, T. Brecht, Y. Y. Gao, S. Shankar, M. Hatridge, G. Catelani et al., NonPoissonian Quantum Jumps of a Fluxonium Qubit due to Quasiparticle Excitations, Phys. Rev. Lett. 113, 247001 (2014).

[44] C. Wang, Y. Y. Gao, I. M. Pop, U. Vool, C. Axline, T. Brecht, R. W. Heeres, L. Frunzio, M. H. Devoret, G. Catelani et al., Measurement and Control of Quasiparticle Dynamics in a Superconducting Qubit, Nat. Commun. 5, 5836 (2014).

[45] D. G. Ferguson, A. A. Houck, and J. Koch, Symmetries and Collective Excitations in Large Superconducting Circuits, Phys. Rev. X 3, 011003 (2013).

[46] G. Viola and G. Catelani, Collective Modes in the Fluxonium Qubit, Phys. Rev. B 92, 224511 (2015). 
[47] P. V. Klimov, J. Kelly, Z. Chen, M. Neeley, A. Megrant, B. Burkett, R. Barends, K. Arya, B. Chiaro, Yu Chen et al., Fluctuations of Energy-Relaxation Times in Superconducting Qubits, Phys. Rev. Lett. 121, 090502 (2018).

[48] D. P. Pappas, M. R. Vissers, D. S. Wisbey, J. S. Kline, and J. Gao, Two Level System Loss in Superconducting Microwave Resonators, IEEE Trans. Appl. Supercond. 21, 871 (2011).

[49] L. DiCarlo, J. M. Chow, J. M. Gambetta, L. S. Bishop, B. R. Johnson, D. I. Schuster, J. Majer, A. Blais, L. Frunzio, S. M. Girvin et al., Demonstration of Two-Qubit Algorithms with a Superconducting Quantum Processor, Nature (London) 460, 240 (2009).

[50] M. W. Johnson, M. H. S. Amin, S. Gildert, T. Lanting, F. Hamze, N. Dickson, R. Harris, A. J. Berkley, J. Johansson, P. Bunyk et al., Quantum Annealing with Manufactured Spins, Nature (London) 473, 194 (2011).

[51] C. Rigetti, J. M. Gambetta, S. Poletto, B. L. T. Plourde, J. M. Chow, A. D. Córcoles, J. A. Smolin, S. T. Merkel, J. R. Rozen, G. A. Keefe et al., Superconducting Qubit in a Waveguide Cavity with a Coherence Time Approaching 0.1 ms, Phys. Rev. B 86, 100506(R) (2012).

[52] T. M. Hazard, A. D. P. A. Gyenis, A. Di Paolo, A. T. Asfaw, S. A. Lyon, A. A. H. A. Blais, and A. A. Houck, Nanowire Superinductance Fluxonium Qubit, Phys. Rev. Lett. 122, 010504 (2019).

[53] W. Zhang, K. Kalashnikov, W.-S. Lu, P. Kamenov, T. DiNapoli, and M.E. Gershenson, Microresonators
Fabricated from High-Kinetic-Inductance Aluminum Films, Phys. Rev. Applied 11, 011003 (2019).

[54] L. Grünhaupt, M. Spiecker, D. Gusenkova, N. Maleeva, S. T. Skacel, I. Takmakov, F. Valenti, P. Winkel, H. Rotzinger, A. V. Ustinov et al., Granular Aluminum: A Superconducting Material for High Impedance Quantum Circuits, Nat. Mater. 18, 816 (2019).

[55] A. Shearrow, G. Koolstra, S. J. Whiteley, N. Earnest, P. S. Barry, F. J. Heremans, D. D. Awschalom, E. Shirokoff, and D. I. Schuster, Atomic Layer Deposition of Titanium Nitride for Quantum Circuits, arXiv:1808.06009, https://doi .org/10.1063/1.5053461.

[56] D. Niepce, J. Burnett, and J. Bylander, High Kinetic Inductance NbN Nanowire Superinductors, Phys. Rev. Applied 11, 044014 (2019).

[57] G. Ithier, E. Collin, P. Joyez, P. J. Meeson, D. Vion, D. Esteve, F. Chiarello, A. Shnirman, Y. Makhlin, J. Schriefl, and G. Schön, Decoherence in a Superconducting Quantum Bit Circuit, Phys. Rev. B 72, 134519 (2005).

[58] R. J. Schoelkopf, A. A. Clerk, S. M. Girvin, K. W. Lehnert, and M. H. Devoret, Qubits as Spectrometers of Quantum Noise, in Quantum Noise in Mesoscopic Physics, edited by Y. V. Nazarov (Springer, Dordrecht, 2003), pp. 175-203.

[59] J. Gambetta, A. Blais, D. I. Schuster, A. Wallraff, L. Frunzio, J. Majer, M. H. Devoret, S. M. Girvin, and R. J. Schoelkopf, Qubit-Photon Interactions in a Cavity: Measurement-Induced Dephasing and Number Splitting, Phys. Rev. A 74, 042318 (2006). 